\title{
Evolution active du tarif par le dépôt de propositions
}

\section{Mirjam Rufer}

Dre méd., cheffe adjointe de la division Médecine et tarifs hospitaliers, FMH

Les procédures de demandes de SwissDRG SA et de l'Office fédéral de la statistique (OFS) permettent de perfectionner les structures tarifaires hospitalières. Toutes deux constituent des piliers essentiels pour participer activement à l'évolution du tarif et visent une rémunération adaptée aux prestations. La FMH propose un soutien avisé à ses sociétés de discipline et organisations faîtières qui souhaitent déposer des demandes.

Les systèmes tarifaires hospitaliers sont des systèmes évolutifs et un élément important de cette évolutivité réside dans les procédures de demandes pour SwissDRG, TARPSY et ST Reha. Dans ces colonnes, nous illustrons le processus à suivre en présentant l'exemple des soins somatiques aigus. Le système de forfaits par cas de SwissDRG se fonde sur le codage, d'une part, des diagnostics selon la version GM (German Modification) de la CIM-10 et, d'autre part, des traitements selon la Classification suisse des interventions chirurgicales (CHOP). La mise à jour et la publication de cette dernière relèvent du domaine de compétence de l'OFS.

La rémunération tarifaire doit s'adapter à la pratique clinique et non l'inverse.

La CHOP sert de base aux structures tarifaires hospitalières et, à ce titre, elle doit reproduire les traitements de manière différenciée pour qu'ils puissent être attribués à un groupe de cas et donc à un forfait adapté.

\section{Valeurs de rémunération recalculées chaque année}

Un code extrêmement détaillé et différencié en fonction de toutes les variantes de traitements possibles impliquerait une explosion de la classification CHOP sans pour autant conduire forcément à une rémunération plus précise de chaque cas. Avec le système tarifaire hospitalier, à l'inverse du tarif ambulatoire à la prestation, les cas médicalement et économiquement comparables sont attribués à un même groupe, duquel découle un forfait. Etablie sur la base des données de prestations et de coûts saisies par les hôpitaux, la va- leur de rémunération de ce forfait par cas est recalculée chaque année.

\section{Système tarifaire en phase avec le quotidien clinique}

Pour que la structure tarifaire soit évolutive et reste adaptée aux prestations fournies, il faut que le système soit constamment perfectionné et les informations sur lesquelles il repose mises à jour. Chaque année, les procédures de demandes de l'OFS et de SwissDRG SA permettent de recueillir le point des vue des utilisateurs, de compléter les structures et ainsi d'aligner le système aux évolutions cliniques.

\section{Soutien actif de la FMH aux sociétés de discipline et aux organisations faîtières}

La FMH fait partie des organisations pouvant déposer des demandes (cf. tab. 1) même si concrètement, ce sont les sociétés de discipline ou les organisations faîtières ${ }^{1}$, et plus exactement un membre de leur présidence ou de leur délégation en charge des DRG, qui les déposent. En revanche, nous sommes à leur entière disposition pour les accompagner et les conseiller dans ce processus. Chaque organisation médicale de la FMH dispose d'un interlocuteur dédié de notre division, chargé de traiter leurs propositions et de les soutenir activement dans la préparation des demandes. Jusqu'à présent, pas moins de 3000 demandes visant à développer la CHOP et les structures tarifaires hospitalières ont été déposées en collaboration avec la division Médecine et tarifs hospitaliers de la FMH. L'énorme engagement de toutes les personnes concernées a permis au corps mé- 
Tableau 1: Détail du processus.

\begin{tabular}{|c|c|c|}
\hline Important à savoir & $\begin{array}{l}\text { Procédure de } \\
\text { demande }\end{array}$ & Description \\
\hline $\begin{array}{l}\text { Organisations pou- } \\
\text { vant déposer des } \\
\text { demandes }\end{array}$ & OFS & $\mathrm{FMH}, \mathrm{H}+$, cantons (CDS), assureurs-maladie et assureurs-accidents, SwissDRG SA \\
\hline Rôle de la FMH & $\begin{array}{l}\text { OFS et } \\
\text { SwissDRG SA }\end{array}$ & $\begin{array}{l}\text { En tant qu'organisation faîtière, la FMH peut déposer des demandes, mais ne le fait } \\
\text { pas. Avec ses expert-e-s, la division Médecine et tarifs hospitaliers de la FMH } \\
\text { conseille et assiste les organisations médicales* qui veulent en déposer. Elle } \\
\text { défend leur point de vue dans les commissions en vue de leur mise en œuvre } \\
\text { auprès de l'OFS. }\end{array}$ \\
\hline $\begin{array}{l}\text { Rôle des organisa- } \\
\text { tions médicales* }\end{array}$ & $\begin{array}{l}\text { OFS et } \\
\text { SwissDRG SA }\end{array}$ & $\begin{array}{l}\text { Les organisations médicales*, représentées par leur présidence ou délégation DRG, } \\
\text { déposent des demandes et contribuent ainsi à l'évolution/au perfectionnement du } \\
\text { système tarifaire et de la classification CHOP dans leurs disciplines respectives. }\end{array}$ \\
\hline $\begin{array}{l}\text { Marche à suivre } \\
\text { pour les demandes } \\
\text { concernant plusieurs } \\
\text { disciplines }\end{array}$ & OFS & $\begin{array}{l}\text { Il est primordial que les organisations médicales* concernées, représentées par } \\
\text { leur présidence ou délégation DRG, se concertent avant le dépôt d'une demande. } \\
\text { Les demandes consolidées peuvent être plus rapidement mises en œuvre par } \\
\text { I'OFS. }\end{array}$ \\
\hline
\end{tabular}

* Sociétés de discipline et organisations faîtières de la FMH

dical d'apporter une contribution importante au processus adaptif et évolutif des systèmes tarifaires.

\section{Recherche d'un critère commun pour la séparation des coûts}

SwissDRG SA utilise exclusivement les données collectées pour faire évoluer les structures tarifaires. Si un collectif de patients déterminé n'est plus couvert par un forfait conformément aux coûts qu'il engendre, il est alors préférable de le séparer des autres cas du même forfait (DRG) en identifiant un critère commun (des données) à tous les patients du collectif concerné. Il peut s'agir par exemple du diagnostic, du traitement, de leur combinaison, du niveau de complexité des diagnostics secondaires (PCCL - Patient Clinical Complexity Level) ou autres. Au même titre que les rémuné- rations supplémentaires ${ }^{2}$ ou la saisie de médicaments ou de substances pour la statistique médicale ${ }^{3}$, les critères qui viennent d'être évoqués peuvent faire l'objet d'une procédure de demande (cf. tab. 2).

\section{Les propositions interdisciplinaires favorisent une mise en œuvre rapide}

Lorsqu'une proposition est remise à l'OFS, il est indispensable de vérifier si le traitement concerné peut être entièrement reproduit par les plus de 14000 codes que compte la CHOP actuellement. Si c'est le cas et que la proposition demande un niveau de différenciation plus élevé pour un code $\mathrm{CHOP}$, seule une différence importante en termes de coûts ou de temps de travail investi permettrait de la justifier. Celle-ci devrait par ailleurs présenter le potentiel, lors d'une prochaine

Tableau 2: Contenus de la procédure de demande, SwissDRG SA (exemple somatique aiguë).

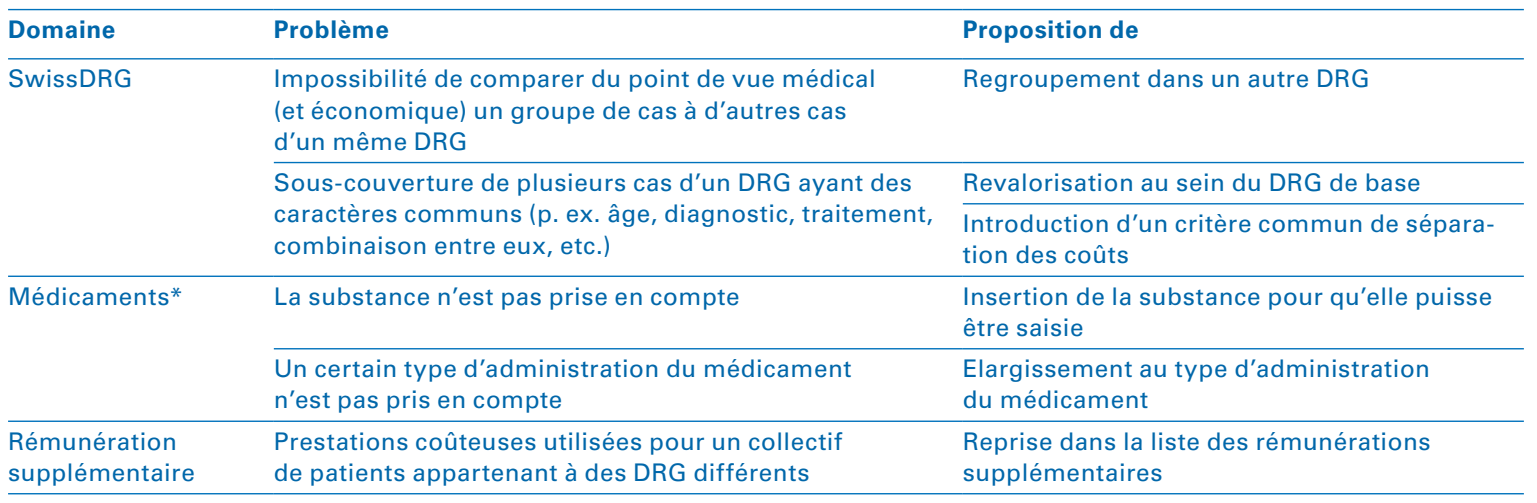

2 Les rémunérations

supplémentaires

s'ajoutent aux forfaits Elles s'appliquent aux procédures, implants, produits sanguins ou médicaments onéreux.

3 Liste des médicaments/ substances à relever dans la statistique médicale des hôpitaux, OFS supplémentaire de patients appartenant à des DRG différents

* Liste des médicaments/substances à relever dans la statistique médicale des hôpitaux

(www.swissdrg.org $\rightarrow$ Somatique aiguë $\rightarrow$ Relevé de données $\rightarrow$ Statistique médicale). 
Tableau 3: Contenus de la procédure de demande, Office fédéral de la statistique.

\begin{tabular}{|c|c|c|}
\hline Domaine & Problème & Proposition de \\
\hline & $\begin{array}{l}\text { Technique/exécution nouvelle/particulière/autre avec différence } \\
\text { de travail ou de coûts considérables par rapport au traitement } \\
\text { actuellement reproduit dans le tarif }\end{array}$ & spécification, différenciation \\
\hline & Traitement ne correspondant pas (plus) à la pratique clinique & suppression \\
\hline & Application pas claire & spécification \\
\hline & Conduit les cas concernés dans un DRG inadéquat & modification, suppression \\
\hline
\end{tabular}

étape, d'être redistribuée au niveau du système de forfaits par cas. Le tableau 3 décrit les contenus possibles des demandes pouvant être déposées à l'OFS. Lorsqu'une proposition concerne plusieurs organisations, il est naturellement pertinent de se concerter. Pour l'OFS, le soutien technique et spécialisé des organisations médicales concernées est indispensable pour la mise en œuvre de la proposition. La concertation préalable des organisations permet de ce fait la mise en œuvre plus rapide d'une proposition.

\section{Anticipation, maître-mot d'une structure tarifaire moderne}

Concernant la CHOP, il faut compter au moins cinq ans entre le dépôt d'une demande et son intégration dans le système tarifaire, afin de pouvoir tenir compte des données. Pour les demandes adressées à SwissDRG SA, cela signifie laisser passer une version et attendre la version tarifaire suivante avant qu'elles puissent être intégrées. Ce délai d'attente est lié au fait que chaque nouvelle version tarifaire est établie à chaque fois sur la base des données annuelles de l'année X-3; l'ensemble du processus d'approbation ne permettant pas d'accélérer cette procédure. Dans ce contexte, il est donc très important de veiller attentivement aux évolutions cliniques afin de les anticiper et de se servir de manière proactive de l'évolutivité du système pour qu'elles soient reprises suffisamment tôt dans le tarif.

\section{Anticipation et coopération permettent la prise en compte de la médecine moderne par le système tarifaire.}

Conclusion: pour un membre d'une société de discipline qui entend déposer une demande, le mieux est de s'adresser à la personne de son organisation en charge des DRG. La FMH se met volontiers à disposition en tenant compte des délais (cf. tab. 4) pour établir une demande ou pour coordonner les échanges interdisciplinaires si d'autres sociétés médicales sont concernées. La procédure de demande nous permet de poursuivre un objectif commun: un tarif qui prend mieux en compte les traitements et un système tarifaire offrant ainsi une rémunération adaptée aux prestations.
FMH

Division Médecine et tarifs hospitaliers

Baslerstrasse 47

CH-4600 Olten

Tél. 0313591111

Fax 0313591112

tarife.spital[at]fmh.ch

Tableau 4: Délais actuels des procédures de demandes.

\begin{tabular}{lll}
\hline A qui? & Sur quels sujets? & Délai 2021 \\
\hline $\begin{array}{lll}\text { Office fédéral de } \\
\text { la statistique }\end{array}$ & CHOP 2023 & $31.5-12.9 .2021$ \\
\cline { 2 - 3 } SwissDRG SA & Manuel de codage 2023 & $31.5-11.7 .2021$ \\
\hline SwissDRG: DRG, rémunérations supplémentaires, médicaments* & $31.5-11.7 .2021$
\end{tabular}

* Liste des médicaments/substances à relever dans la statistique médicale des hôpitaux

(www.swissdrg.org $\rightarrow$ Somatique aiguë $\rightarrow$ Relevé de données $\rightarrow$ Statistique médicale). 